\title{
Isothermal Crystallization of Blends Containing Two Thermotropic Liquid Crystalline Polymers
}

Chen Qian ${ }^{\mathrm{a}}$, Guigui Wan ${ }^{\mathrm{b}}$, Charles E. Frazier ${ }^{\mathrm{b}}$, Donald G. Baird ${ }^{\mathrm{a}^{*}}$,

${ }^{a}$ Department of Chemical Engineering, Virginia Polytechnic Institute and State University, Blacksburg, VA 24061

${ }^{\mathrm{b}}$ Department of Sustainable Biomaterials, Virginia Polytechnic Institute and State University, Blacksburg, VA 24061

\section{Abstract}

The intention of this work is to determine how the isothermal crystallization behavior of a thermotropic liquid crystalline polymer (TLCP) can be adjusted by blending it with a pure TLCP of lower melting point. One TLCP $\left(\mathrm{T}_{\mathrm{m}} \sim 350^{\circ} \mathrm{C}\right)$ used is a copolyester synthesized from terephthalic acid, 4-hydroxybenzoic acid, hydroquinone and hydroquinone derivatives. The other TLCP $\left(\mathrm{T}_{\mathrm{m}} \sim 280{ }^{\circ} \mathrm{C}\right)$ is a copolyesteramide of $60 \mathrm{~mol} \%$ hydroxynaphthoic acid, $20 \mathrm{~mol} \%$ terephthalic acid and $20 \mathrm{~mol} \%$ 4-aminophenol. As the content of the low melting TLCP increases in the blends, the temperature at which isothermal crystallization occurs decreases. Comparing with pure TLCPs, the blend of $75 \%$ low melting TLCP crystallizes at a lower temperature than the pure matrix, and the blend remains as a stable super-cooled fluid in the temperature range from 220 to $280^{\circ} \mathrm{C}$ for 1200 s. Because of the low energy released during the phase transition, small amplitude oscillatory shear (SAOS) is more sensitive to detecting the onset of isothermal crystallization than differential scanning calorimetry (DSC).

Keywords: Thermotropic liquid crystalline polymers blends, isothermal crystallization, rheology 


\section{Introduction}

Thermotropic liquid crystalline polymers (TLCPs) have attracted great research interests because of the combination of their promising properties, which includes high stiffness and strength, low permeability to gas molecules, low linear thermal expansion coefficient, reduced dielectric constants and outstanding chemical resistance [1-5]. Because of these properties, TLCPs have been used in various applications, such as the reinforcing components in TLCP/thermoplastic blends, barrier material for packaging, as well as electrical parts [4, 6-9]. TLCPs also show promise in the emerging fields, including high pressure gas storage and fused filament fabrication [10-12].

Because of the limited types of commercial TLCPs available, researchers have blended two TLCPs to expand the potential applications of TLCPs. The TLCP/TLCP blends have demonstrated distinctive properties relative to the neat components. One important character possessed by the blends is the improved mechanical properties [13-17]. For example, Vallejo et al. [13] injection molded the blends containing two TLCPs, which were the 20/80 poly(ethylene terephthalate) (PET)/hydroxybenzoic acid (HBA) and 73/27 HBA/2,6-hydroxynaphthoic acid (HNA). The modulus and strength for the 20/80 PET/HBA material were $9.8 \mathrm{GPa}$ and $175 \mathrm{MPa}$, respectively. For the 73/27 HBA/HNA copolyester, the modulus and strength were $9.5 \mathrm{GPa}$ and $210 \mathrm{MPa}$, respectively. For the blend with $80 \%$ TLCP of HBA/HNA, the modulus was $1.18 \mathrm{GPa}$, and the strength was $300 \mathrm{MPa}$. Both values were greater than those of either pure TLCP components.

Depending on the miscibility and the chemical composition of the materials, the TLCP/TLCP blends also exhibit lower viscosity relative to each of the two neat resins, which 
could be beneficial for processing [14, 18-19]. Kenig et al. [14] blended two TLCPs using a single screw extruder. The two TLCPs used were the 73/27 HBA/HNA and a copolymer composed of $60 \mathrm{~mol} \%$ HNA, $20 \mathrm{~mol} \%$ terephthalic acid (TA), and 20 mol\% acetoxy-acid aniline, respectively. The viscosity of the blends was measured in the shear rate range of 10 to $10^{4} \mathrm{~Pa}^{-1}{ }^{-1}$ with a capillary rheometer. Within this a shear rate range, the viscosity curves of the blends containing 25, 50, and 75\% HBA/HNA copolymer nearly overlapped. The viscosity of these blends was lowered than that of either of the neat components, especially in the shear rate range of 10 to $10^{2} \mathrm{~Pa}^{-1} \mathrm{~s}^{-}$Lin and Winter [18] measured the viscosity of the blends containing the copolymers of 73/27 HBA/HNA and 40/60 PET/HBA at the shear rates from 0.05 to $0.4 \mathrm{~s}^{-1}$. The viscosity of the blends was lower than that of the neat materials. It was interesting to observe that with the addition of $10 \mathrm{wt} \%$ 40/60 PET/HBA, the viscosity of the blend was reduced by a factor of 4 relative to the neat 73/27 HBA/HNA. Heish et al. [19] speculated the lower viscosity of the blends was due to the enhanced orientation, as well as the lubrication between the two different TLCP molecules.

The thermal properties of the blends containing two TLCPs with the same chemical moiety also vary from those of the pure materials. McLeod and Baird [20] reported the melting and crystallization temperatures of a TLCP can be tailored by blending it with a second TLCP of lower melting point. Both TLCPs used in this work were synthesized from HBA, terephthalic acid (TA), hydroquinone and hydroquinone derivatives, but the monomer ratios were different for the two TLCPs. The TLCP containing a greater amount of HBA exhibited a higher melting point of around $330^{\circ} \mathrm{C}$, while the other TLCP melted at about $272^{\circ} \mathrm{C}$. One intriguing observation was that the crystallization temperature of the blends was a linear function of the ratio of the two 
TLCPs. The lower melting and crystallization temperatures of the TLCP/TLCP blends allowed them to be combined with thermoplastics possessing lower processing temperatures.

McLeod and Baird's work focused on the effect of the cooling rate on the crystallization temperatures of the TLCP/TLCP melts. However, to process TLCP/TLCP blends in the supercooled state, knowing the melting and crystallization temperatures from the temperature ramp experiments is not enough. The isothermal crystallization behavior at different temperatures below the melting point needs to be investigated to guide processing. The main objective of this work is to determine how the isothermal crystallization behavior of a TLCP can be adjusted by blending it with another TLCP of lower melting temperature. In this work the TLCP/TLCP blends and neat resins are fully melted first, and then cooled down to a constant temperatures below the melting point. The isothermal crystallization is monitored using both dynamic mechanical and DSC measurements. In addition to this focus, the sensitivities of the two approaches on detecting isothermal crystallization are compared. The practical significance of this work is to tailor the super-cooling behavior of a high melting TLCP, such that it can be blended with thermoplastic of lower processing temperatures. 


\section{Experimental}

\subsection{Materials}

Two commercial TLCPs were used in this study. The first TLCP was provided by Dupont with a commercial name of Zenite HX6000. It is a liquid crystalline copolyester synthesized from hydroxybenzoic acid (HBA), terephthalic acid (TA), hydroquinone (HQ) and hydroquinone derivatives (HQ-derivatives). However, the monomer ratio for this material is unknown. It has a density of $1.38 \mathrm{~g} / \mathrm{cm}^{3}$, and it is usually processed at temperatures of $360^{\circ} \mathrm{C}$ and above. In this report this TLCP will be designated as TLCP-A.

The second TLCP was supplied by Ticona-Celanese, and its commercial name is Vectra B950. Vectra B950 is a copolyesteramide of 60 mol\% 2,6-hydroxynaphthoic acid (HNA), 20 mol\% (TA), and 20 mol\% p-amino-phenol (PA). Compared with Zenite HX6000, Vectra B950 has a lower melting point of around $280^{\circ} \mathrm{C}$. Vectra $\mathrm{B} 950$ will be referred to as TLCP-B in later discussion. Both TLCPs were used as received.

\subsection{Melt compounding of the TLCP/TLCP blends}

The pellets of the two TLCP resins were mixed by tumbling in a container for 5 min, with the weight ratios of $25 / 75,50 / 50$ and $75 / 25$, respectively. The mixed pellets as well as the neat resins were dried in a vacuum oven at $100{ }^{\circ} \mathrm{C}$ for at least $24 \mathrm{hr}$ before use. A Killion KL-100 single screw extruder with a $1.59 \mathrm{~mm}$ diameter capillary die was used for melt compounding. A Maddock screw with $25.4 \mathrm{~mm}$ diameter and 30:1 L:D was employed. The temperatures from zone 1 to zone 4 were set at $300,340,350$ and $360^{\circ} \mathrm{C}$, respectively. The screw speed was $30 \mathrm{rpm}$. After being plasticated in the extruder, the polymer melt was quenched in a cold water bath. The 
obtained polymer strands were then chopped into pellets, using a Killion Model 2" pelletizer. The pellets were used for rheological and thermal analysis.

\subsection{Differential Scanning Calorimetry}

DSC measurements in the temperature ramp mode were first perfomed using a TA instrument's Discovery DSC. The temperature ramp tests were used to obtain the melting points, as well as the crystallization temperatures of the TLCP/TLCP blends and the neat materials. Ca. $10 \mathrm{mg}$ of each samples was first equilibrated at $40{ }^{\circ} \mathrm{C}$, then the temperature was increased to $360^{\circ} \mathrm{C}$. After equilibration at $360^{\circ} \mathrm{C}$ for $1 \mathrm{~min}$, the materials were cooled to $40^{\circ} \mathrm{C}$ (first cooling scan), and then heated back up to $360^{\circ} \mathrm{C}$ (second heating scan). A constant $10^{\circ} \mathrm{C} / \mathrm{min}$ temperature cycle was applied in all the heat/cool/heat cycles, and all measurements were performed under a dry nitrogen environment. The results were found to be independent of the equilibration time at $360^{\circ} \mathrm{C}$. Therefore, $1 \mathrm{~min}$ equilibration time guaranteed the complete melting of the materials. The melting point as well as the melting enthalpy were calibrated using indium.

To investigate the isothermal crystallization of the materials, DSC tests were also carried out in the isothermal time sweep mode. In this case the materials were first equilibrated at $360^{\circ} \mathrm{C}$ for $1 \mathrm{~min}$, then the temperature was lowered to the predetermined values below the melting points at a rate of $10^{\circ} \mathrm{C} / \mathrm{min}$. The heat flow was tracked as a function of time for 40 mins.

\subsection{Small amplitude oscillatory shear measurements}

Small amplitude oscillatory shear (SAOS) measurements were performed in the temperature ramp mode to obtain the crystallization temperatures, using a TA instrument's ARES-G2 rheometer. The pellets of the melt compounded TLCP/TLCP blends and the neat resins were compression molded into disks at $360^{\circ} \mathrm{C}$. All samples were dried at $100^{\circ} \mathrm{C}$ under 
vacuum for at least $24 \mathrm{hr}$ prior to testing. $25 \mathrm{~mm}$ parallel disks fixtures with $1 \mathrm{~mm}$ gap were used. Each sample was first equilibrated at $360^{\circ} \mathrm{C}$ for $3 \mathrm{~min}$, then the temperature was decreased at a rate of $10^{\circ} \mathrm{C} / \mathrm{min}$. Meanwhile dynamic shear with $5 \%$ strain and $10 \mathrm{rad} / \mathrm{s}$ angular frequency was applied. The storage modulus G' and loss modulus G" were recorded as a function of time. All experiments were conducted under a dry nitrogen environment.

SAOS was also used to investigate the isothermal crystallization of the TLCP/TLCP blends as well as the neat resins in the super-cooled state. All materials were first equilibrated at $360^{\circ} \mathrm{C}$ for 3 mins, such that the exclusion of crystalline structure was ensured. Then the materials were cooled to different designated supercooling temperatures below the melting points at $10^{\circ} \mathrm{C} / \mathrm{min}$. G' and G" were monitored as a function of time while the samples were sheared at 10 $\mathrm{rad} / \mathrm{s}$ frequency and $5 \%$ strain.

To investigate the effect of strain on the isothermal crystallization rate in SAOS measurements, after being equilibrated at $360^{\circ} \mathrm{C}$, each sample was cooled to a temperature below the melting point. SAOS was applied with a constant angular frequency, $\omega$, of $10 \mathrm{rad} / \mathrm{s}$, and the strain used was 1, 2 and 5\%, respectively. The crossover time of G' and G" was recorded, and the values obtained from different strain amplitudes were compared.

\section{Results and Discussion}

\subsection{DSC heat/cool/heat scans}

Figs 1 (a) and (b) show the results of DSC second heating and first cooling scans, respectively. In the heating scan the melting point of the material is defined as the temperature at which all crystalline structures are excluded. Therefore, the melting point refers to the hightemperature end of the melting endotherm, as indicated by the arrows in Fig 1 (a). In the cooling 
scan, the onset and peak crystallization temperatures are extracted, as represented in Fig 1 (b). The melting and crystallization enthalpies obtained from Fig 1 are summarized in Table 1.

It can be observed that both the melting and crystallization temperatures of the TLCP-A are lowered when it is blended with the TLCP-B. It should be noticed that the TLCP-B exhibits repeatable double melting peaks in Fig 1 (a). Therefore, the uppermost temperature of the higher melting peak is designated as the melting point for TLCP-B, as indicated by the arrow in Fig 1 (a). The melting point is decreased from $349{ }^{\circ} \mathrm{C}$ for the neat TLCP-A to 341 and $308^{\circ} \mathrm{C}$ for the blends containing 25 and 50\% of TLCP-B, respectively. For the 75\% TLCP-B blend, no melting point was observed from the heating scan. Additionally, the TLCP/TLCP blends also exhibit lower onset and peak crystallization temperatures relative to the neat TLCP-A. For example, the onset crystallization temperature of the pure TLCP-A is decreased from $301^{\circ} \mathrm{C}$ to 278 and $247^{\circ} \mathrm{C}$ for the blends of 25 and $50 \%$ of TLCP-B, respectively. The most striking case occurs in the blend containing $75 \%$ TLCP-B. Its onset crystallization temperature is $194^{\circ} \mathrm{C}$, which is lower than the onset crystallization temperature of either of the neat TLCPs.

Table 1 shows the addition of the TLCP-B to the TLCP-A decreases both the melting and crystallization enthalpies. It should be noted that the melting and crystallization enthalpies of the blends are even lower than those of the neat resins. For the blend with the 75\% TLCP-B, the enthalpy of crystallization is only $0.8 \mathrm{~J}_{\mathrm{og}}{ }^{-1}$. We suggest the lack of a melting endotherm for this blend in Fig 1 is due to the small amount of energy consumed for melting, which is not detected by DSC.

There are two possible reasons for the unusually low crystallization temperature for the 75\% TLCP-B blend. First, a transesterification reaction can occur in the extruder, when the two 
TLCPs are blended [21]. The product of the reaction might well be a new material with lower crystallization temperature. Second, the melting and crystallization peaks of the blends are single peaks, which are also shifted relative to those of the neat materials. This suggests, but does not verify that the two TLCPs are compatible or possibly miscible. At the particular content of $75 \%$

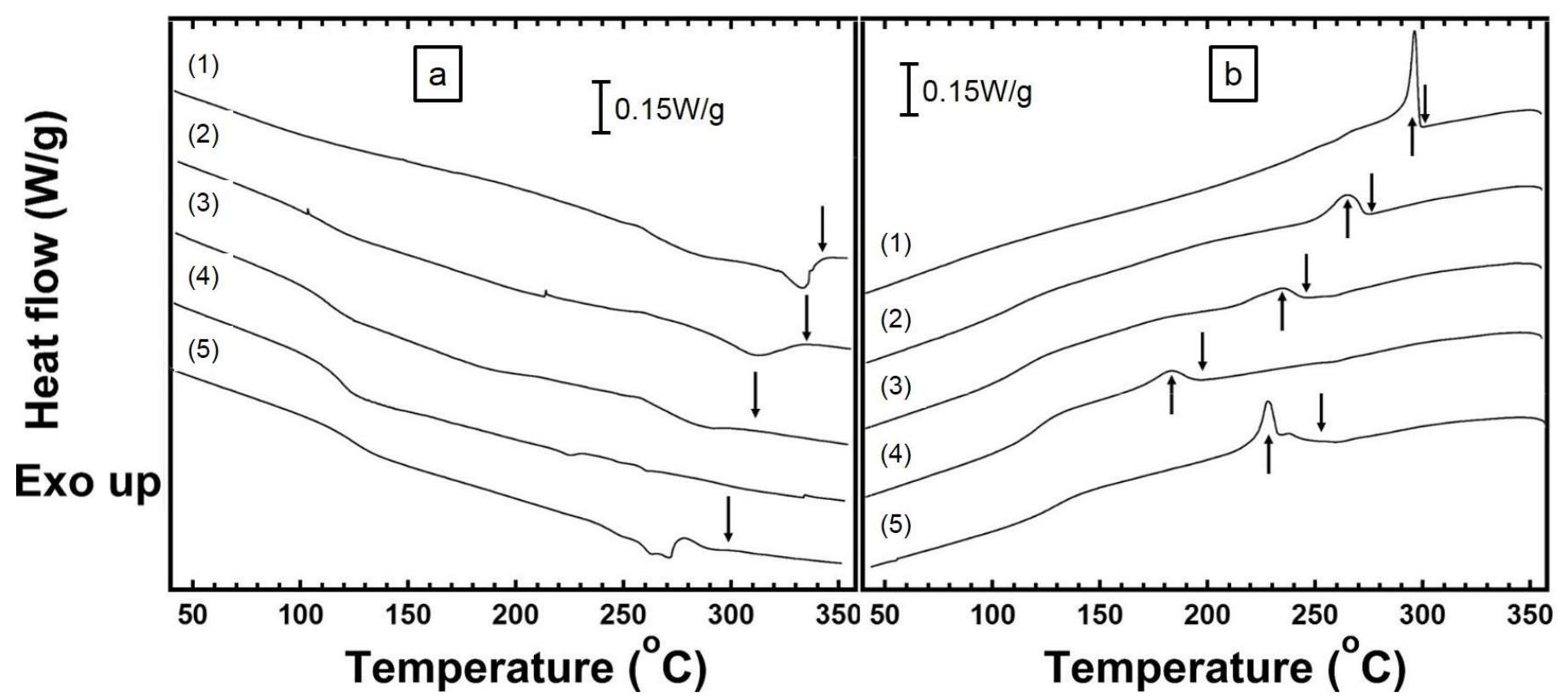

TLCP-B, the two TLCPs may co-crystallize at a lower temperature.

Fig 1: DSC second heating and first cooling scans of the TLCP/TLCP blends, as shown in (a) and (b), respectively. In both figures the weight contents of the low melting TLCP-B from top to bottom are (1) $0 \%$, (2) $25 \%$, (3) $50 \%$, (4) $75 \%$ and (5) $100 \%$. In (a) Arrows indicate the melting points for each material, while in (b) Arrows pointing down indicate the onset crystallization temperatures, arrows pointing up represent the peak crystallization temperatures. The vertical bar suggests the scale in heat flow. 
Table 1: Enthalpies of melting and crystallization obtained from Fig 1.

\begin{tabular}{|c|c|c|}
\hline $\begin{array}{c}\text { Low melting TLCP-B content } \\
/ \mathrm{wt} \%\end{array}$ & Melting enthalpy $/ \mathrm{J} \cdot \mathrm{g}^{-1}$ & ${\text { Crystallization enthalpy } / \mathrm{J} \cdot \mathrm{g}^{-1}}^{-1}$ \\
\hline 0 & 6.5 & 5.2 \\
\hline 25 & 2.9 & 1.7 \\
\hline 50 & 1.0 & 0.8 \\
\hline 75 & N/A & 2.8 \\
\hline 100 & 2.2 & 2.8 \\
\hline
\end{tabular}

\subsection{SAOS cooling ramp}

To complement the crystallization temperatures obtained from DSC, SAOS measurements were carried out, the results are presented in Fig 2. The crossover temperatures of G' and G' are recorded as the crystallization temperatures. For the neat TLCP-A, and the blends containing 25 and 50\% TLCP-B, the crossover temperatures of G' and G' are within the ranges of the onset and peak crystallization temperatures obtained from DSC. For the 75\% TLCP-B blend as well as the neat TLCP-B, the crossover temperatures of G' and G' are closer to the peak crystallization temperatures. Overall, the crystallization temperatures obtained from SAOS are in good agreement with those measured from DSC. 


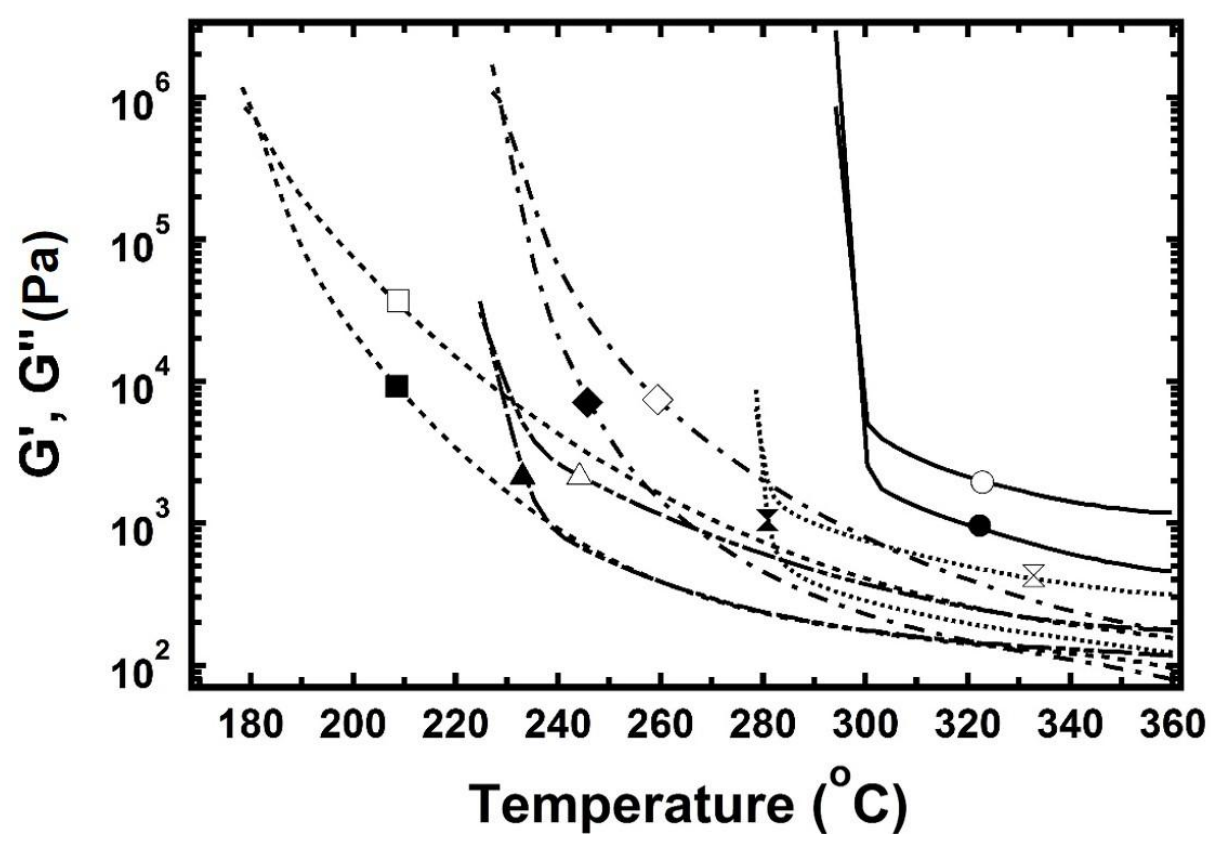

Fig 2: G' and G" as a function of temperature obtained from the SAOS temperature ramp measurements. The symbols represent the content of the TLCP-B as follows: 0\%

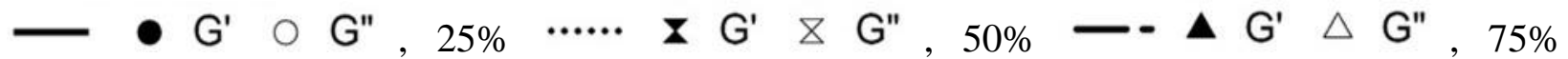
$\ldots \mathrm{G}^{\prime} \square \mathrm{G}^{\prime \prime}$ and $100 \%-\cdot \diamond \mathrm{G}^{\prime} \diamond \mathrm{G}^{\prime \prime}$ 


\subsection{Isothermal crystallization}

Figs 3 (a) and (b) show the isothermal crystallization of the TLCP-A at the temperatures of $330,320,315$ and $310^{\circ} \mathrm{C}$, using the methods of SAOS and DSC. The SAOS results indicate crystallization does not occur at 330 and $320^{\circ} \mathrm{C}$ within 1200s. The crossovers of G' and G" are observed at 847 and $415 \mathrm{~s}$ at the temperature of 315 and $310^{\circ} \mathrm{C}$, respectively. This indicates the crystallization the TLCP is accelerated at lower temperature, as expected. Isothermal DSC also shows no sign of crystallization at 330 and $320^{\circ} \mathrm{C}$. However, the DSC trace is flat at $315^{\circ} \mathrm{C}$. Even though the DSC test is carried out for an extended time of 2400s, still no exothermal peak is observed. At $310^{\circ} \mathrm{C}$, the DSC trace exhibits an exothermic peak, which suggests crystallization. Therefore, the results from SAOS and DSC are at least in qualitative agreement at the temperatures of 330,320 and $310^{\circ} \mathrm{C}$. Nevertheless, DSC does not detect any sign of crystallization at $315^{\circ} \mathrm{C}$, but SAOS does.

The discrepancy between the results obtained from the two methods at $315^{\circ} \mathrm{C}$ can be due to two reasons. One possibility is that the TLCP only releases a small amount of energy during crystallization, and it is not detected by DSC. During the crystallization process, the rigid molecules can only slide pass each other along the chain direction, or rotate along their axes [22]. As a consequence, the crystallization of TLCPs is proposed to be induced by the matching of counits among adjacent molecules, resulting in weak cohesion of chains in the crystal lattice. This process is associated with a low enthalpy change. Strain induced crystallization is another potential reason that introduces the distinctive results obtained from the two techniques. The effect of strain on the crystallization rate in the SAOS measurements will be discussed in detail in $§ 4.4$. 
Although SAOS suggests the occurrence of crystallization at $310^{\circ} \mathrm{C}$, it is challenging to extract detailed information regarding the crystallization kinetics from SAOS. Employing SAOS to track the isothermal crystallization of TLCPs is performed to a much lesser extent relative to DSC. Few have related the dynamic rheological behavior to the physical phenomena associated with the crystallization of TLCPs, neither has any model been proposed to fit the evolution of moduli for TLCPs and TLCP/TLCP blends. As a result, SAOS is more suitable to qualitatively determining the occurrence of a phase transition under isothermal conditions. The practical significance of the rheological measurements is to elucidate how long the TLCPs and their blends can stay fluid in the super-cooled state, and whether processing operation can be carried

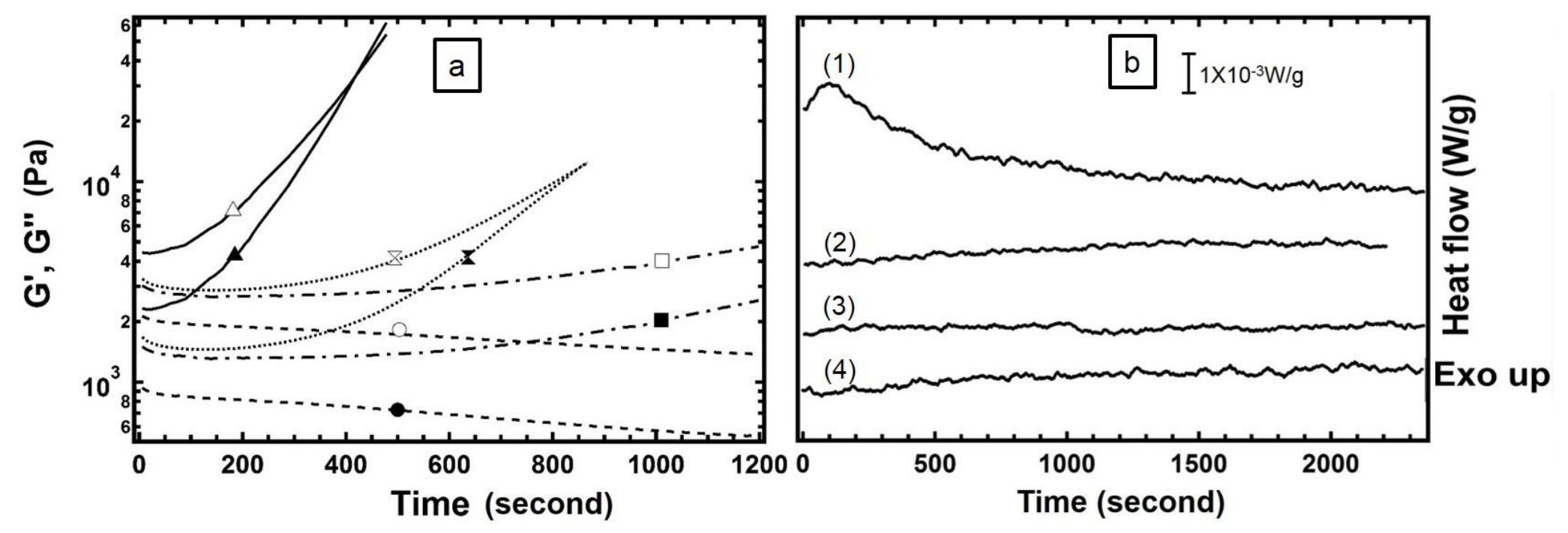

out at given temperatures.

Fig 3: (a) SAOS measurements in the isothermal time sweep mode for neat TLCP-A. The material is first melted at $360^{\circ} \mathrm{C}$, then cooled to the temperatures indicated by the symbols:

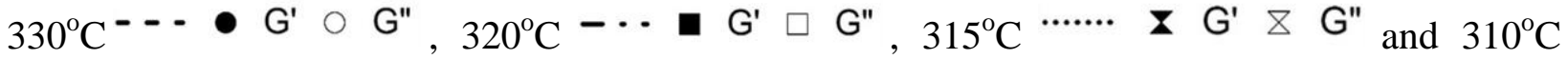
$\Delta G^{\prime} \triangle G^{\prime \prime}$. (b) DSC measurements in the isothermal time sweep mode. The material is 
first melted at $360^{\circ} \mathrm{C}$, then cooled to the predetermined temperatures. From top to bottom are the results obtained at (1) $310^{\circ} \mathrm{C}$, (2) $315^{\circ} \mathrm{C}$, (3) $320^{\circ} \mathrm{C}$ and (4) $330^{\circ} \mathrm{C}$.

The isothermal phase transition of the neat TLCP-B is shown in Fig 4. SAOS results in Fig 4 (a) suggest the TLCP maintain its fluidity within 1200 s at 270 and $260^{\circ} \mathrm{C}$, while crystallization is observed at 250,245 and $240^{\circ} \mathrm{C}$. In Fig 4 (b) DSC also demonstrates the occurrence of crystallization in the temperature range from 240 to $250^{\circ} \mathrm{C}$, but no crystallization exotherm is observed at 270 and $260^{\circ} \mathrm{C}$. Comparing with the neat TLCP-A, better agreement is
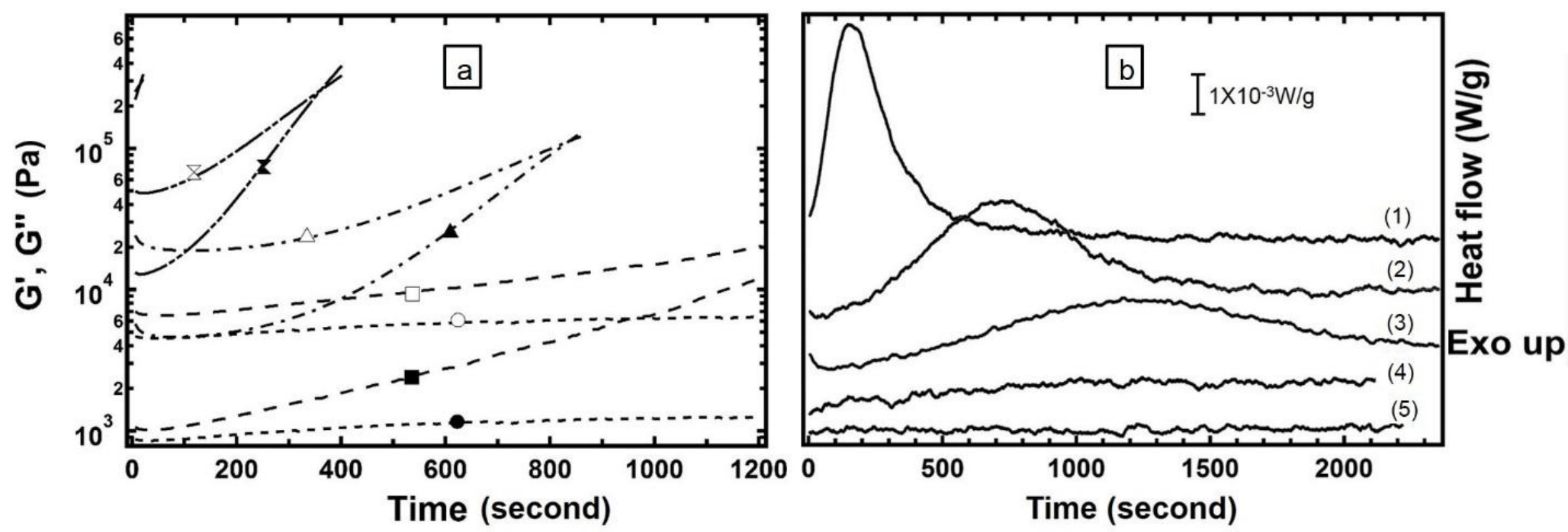

achieved from the SAOS and DSC results for the TLCP-B.

Fig 4: (a) SAOS measurements in the isothermal time sweep mode for the neat TLCP-B. The material is first melted at $360^{\circ} \mathrm{C}$, then cooled to the temperatures indicated by the symbols: $270^{\circ} \mathrm{C}-\cdots \bullet \mathrm{G}^{\prime} \circ \mathrm{G}^{\prime \prime}, 260^{\circ} \mathrm{C}-\cdots \mathrm{G}^{\prime} \square \mathrm{G}^{\prime \prime}, 250^{\circ} \mathrm{C}-\cdots \Delta \mathrm{G}^{\prime} \triangle \mathrm{G}^{\prime \prime}, 245^{\circ} \mathrm{C}$ — $~ G^{\prime}$ \& $G^{\prime \prime}$, and $240^{\circ} \mathrm{C}-$. (b) DSC measurements in the isothermal time sweep mode. 
The material is first melted at $360^{\circ} \mathrm{C}$, then cooled the predetermined temperatures. From top to bottom are the results obtained at (1) $240^{\circ} \mathrm{C}$, (2) $245^{\circ} \mathrm{C}$, (3) $250^{\circ} \mathrm{C}$, (4) $260^{\circ} \mathrm{C}$ and (5) $270^{\circ} \mathrm{C}$.

As the isothermal crystallization results of the blends containing 25 and 50\% TLCP-B are represented in the supplemental materials, now we turn our attention to the most interesting TLCP/TLCP blends, which contains 75\% TLCP-B. This blend demonstrated even lower crystallization temperature than either of the pure component (Figs 1 and 2). The SAOS and DSC results are shown in Fig 5 (a) and (b), respectively. A DSC heating scan fails to identify the melting point of this blend, and an alternative method is adopted, which is the SAOS experiment in the isothermal time sweep mode. In this method the time sweep experiments were performed at $270,280,290$ and $300^{\circ} \mathrm{C}$. The evolutions of G' and G' as a function of time were monitored while oscillatory shear with $10 \mathrm{rad} / \mathrm{s}$ frequency and 5\% strain was applied. It is found that G' and G” gradually increase at 270 and $280^{\circ} \mathrm{C}$. In contrast, they are each constant as time elapses at temperatures higher than $290^{\circ} \mathrm{C}$ (results not represent here). Thus the melting point of the blend is between 280 and $290^{\circ} \mathrm{C}$. As shown in Fig 5 (a), the rheological approach indicates the blend is in a stable super-cooled state in the temperature range from 220 to $230^{\circ} \mathrm{C}$. This temperature range overlaps well with the processing temperatures of some commodity thermoplastics, such as polyolefins. Fig 5 (b) shows the DSC traces for isothermal crystallization. Crystallization can be observed at 200 and $205^{\circ} \mathrm{C}$, which agrees with the SAOS results. Although SAOS also suggests the occurrence of crystallization at $210^{\circ} \mathrm{C}$, the DSC is unable to detect a crystallization exotherm at this temperature. 


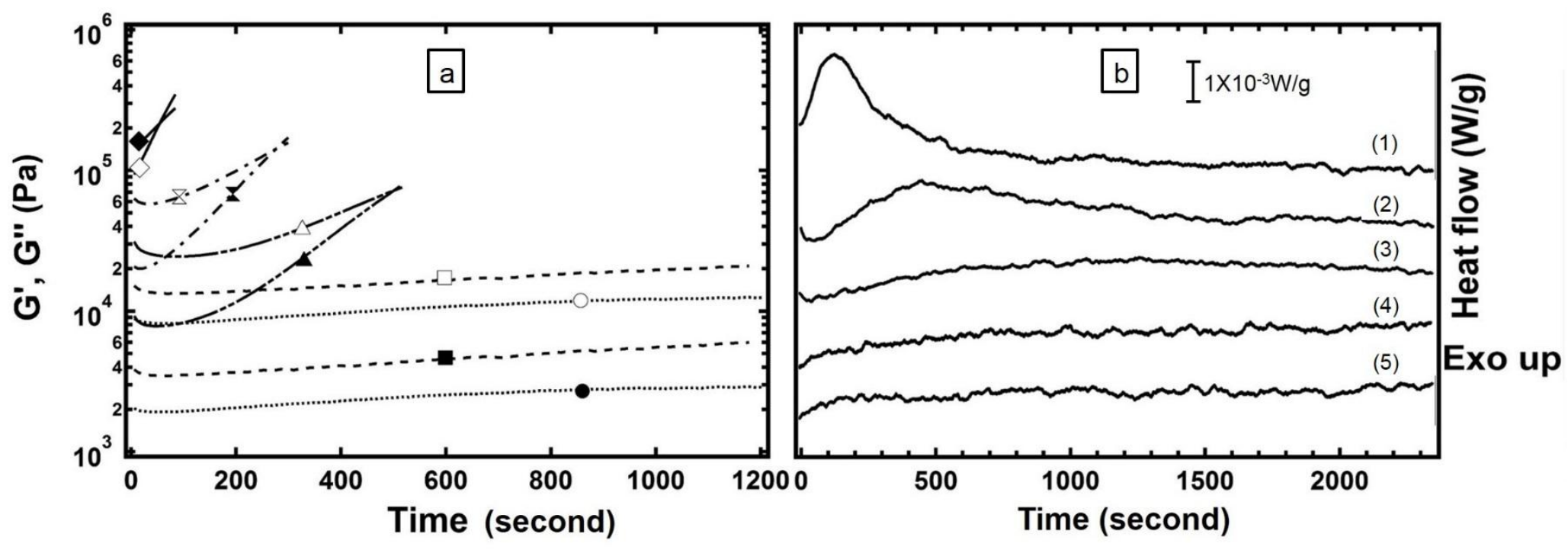

Fig 5: (a) SAOS measurements in the isothermal time sweep mode for the blend containing $75 \%$ TLCP-B. The material is first melted at $360^{\circ} \mathrm{C}$, then cooled to the temperatures indicated by the symbols: $230^{\circ} \mathrm{C} \cdots \cdots \cdot \mathrm{G}^{\prime} \circ \mathrm{G}^{\prime \prime}, 220^{\circ} \mathrm{C}---\square \mathrm{G}^{\prime} \square \mathrm{G}^{\prime \prime}, 210^{\circ} \mathrm{C}$ — $\Delta \mathrm{G}^{\prime} \triangle \mathrm{G}^{\prime \prime}, 205^{\circ} \mathrm{C}-\cdots \mathbf{\text { 又 }} \mathrm{G}^{\prime} \mathrm{\nabla} \mathrm{G}^{\prime \prime}$, and $200^{\circ} \mathrm{C}-\diamond \mathrm{G}^{\prime} \diamond \mathrm{G}^{\prime \prime}$. (b) $\mathrm{DSC}$ measurements in the isothermal time sweep mode. The material is first melted at $360^{\circ} \mathrm{C}$, then cooled to the predetermined temperatures. From top to bottom are the results obtained at (1) $200^{\circ} \mathrm{C}$, (2) $205^{\circ} \mathrm{C},(3) 210^{\circ} \mathrm{C}$, (4) $220^{\circ} \mathrm{C}$ and (5) $230^{\circ} \mathrm{C}$.

\subsection{Effect of strain on the isothermal crystallization rate in SAOS measurements}

Shear-induced crystallization is one possible reason that causes the discrepency between the isothermal crystallization results obtained from SAOS and DSC. Shear-induced crystallization is common among flexible chain polymers like polyethylene and polypropylene [23-26]. The strain applied in the SAOS tests may result in the alignment of TLCP molecules and accelerated crystallization [27-28], such that crystallization will be observed in the dynamic rheological experiments, but not in DSC tests. To investigate whether the strain amplitude applied in SAOS has an impact on the isothermal crystallization rate, the TLCP/TLCP blends 
and neat resins were tested at the predetermined temperatures with $1,2.5$ and $5 \%$ strain, respectively. For each sample, the temperature used was the one at which disagreement in SAOS and DSC results were found. The results are shown in Fig 6. If strain does play an important role on crystallization, we would expect it takes less time for G' and G' to crossover at a higher strain level.

Fig 6 shows the crossover time of G' and G' is generally comparable for different strain amplitude. Higher strain does not necessarily lead to shorter crossover time and faster crystallization. Therefore, the disagreement in the SAOS and DSC reults is believed due the lack of sensitivity of DSC on detecting isothermal crystallization, rather than the strain induced crystallization in SAOS experiments.

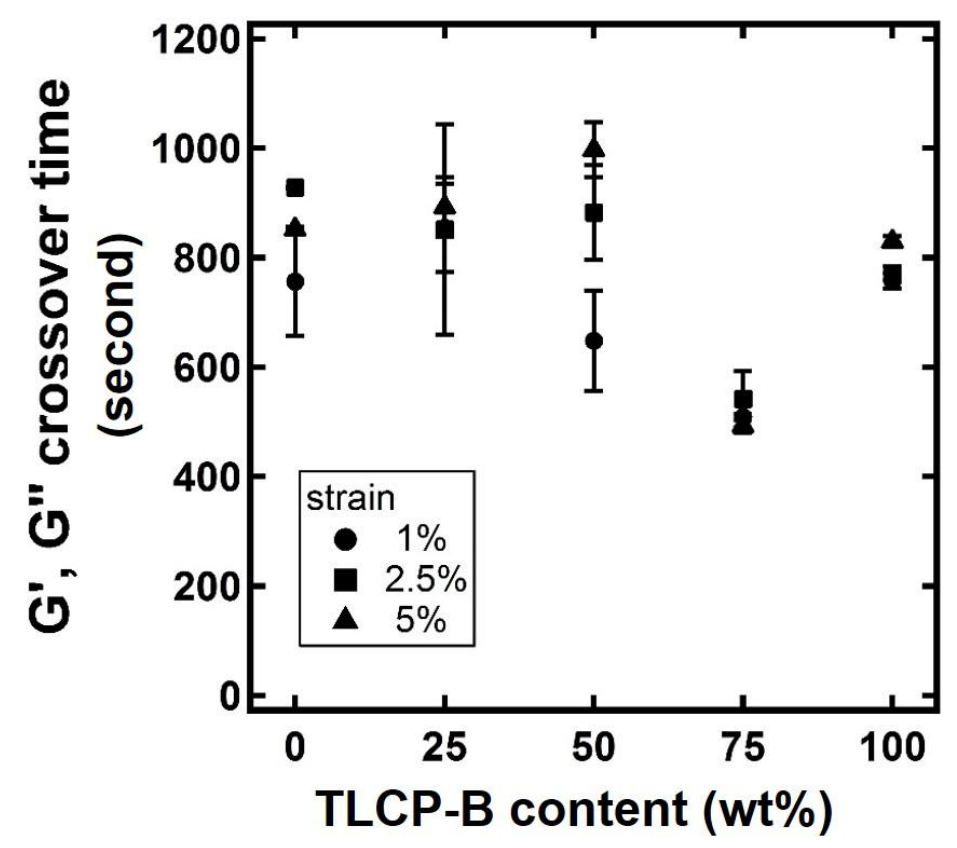

Fig 6: Effect of strain on the crossover time of G' and G' for the TLCP/TLCP blends and the neat TLCPs. All materials are melted in the first place, and then cooled to the predetermined 
temperatures. For different TLCP-B contents, the measurements are performed at the following temperatures: $0 \% 315^{\circ} \mathrm{C}, 25 \% 305^{\circ} \mathrm{C}, 50 \% 255^{\circ} \mathrm{C}, 75 \% 210^{\circ} \mathrm{C}$ and $100 \% 250^{\circ} \mathrm{C}$.

\section{Conclusions}

As the content of the low melting TLCP (TLCP -B) increases in the blends, the temperature at which isothermal crystallization occurs decreases. This suggests relative to the high melting TLCP (TLCP-A), the blends can be potentially processed at lower temperatures in the super-cooled state. The blend containing 75\% TLCP-B is of most interest. In the cooling experiments, it exhibits a lower crystallization temperature relative to both pure TLCPs. Once melted, the isothermal crystallization does not occur unless the material is cooled to $210{ }^{\circ} \mathrm{C}$ or below. The low crystallization temperature allows this blend to be combined with low melting temperature thermoplastics, such as polyolefins.

DSC frequently fails in detecting the isothermal crystallization for both TLCP/TLCP blends and neat TLCPs, which is well captured by SAOS. We believe this is due to the low crystallization enthalpy of the TLCP materials and the TLCP/TLCP blends under isothermal conditions. Therefore, SAOS is more sensitive on characterizing the onset of isothermal crystallization. Although in practical polymer processing, high shear rate and high strain are usually applied, SAOS measurements at least provide the lower temperature limit for the processing of neat TLCP resins and TLCP/TLCP blends in the super-cooled state.

\section{Acknowledgements}

The author would like to thank the Department of Energy through the Savanah River National Laboratories for the financial support. The authors acknowledge Prof. Dr. Garth L. 
Wilkes of Virginia Tech for the tremendous inputs and productive discussions. The authors would also like to thank Ms. Ann Norris in the Department of Sustainable Materials at Virginia Tech for providing assistance on DSC measurements.

\section{References}

[1] H. G. Chae and S. Kumar, "Rigid-rod polymeric fibers", Journal of Applied Polymer Science, vol.100, pp 791-802, 2006.

[2] J. S. Chiou and D. R. Paul, "Gas transport in a thermotropic liquid-crystalline polyester", Journal of Polymer Science Part B: Polymer Physics, vol.25, pp 1699-1707, 1987.

[3] N. R. Miranda, J. T. Willits, B. D. Freeman and H. B. Hopfenberg, "Organic vapor sorption and transport in a thermotropic liquid crystalline polyester", Journal of Membrane Science, vol.94, pp 67-83, 1994.

[4] T.-s. Chung, Thermotropic liquid crystal polymers : thin-film polymerization, characterization, blends, and applications, Technomic Pub. Co., Lancaster, 2001.

[5] A. Donald, A. Windle and S. Hanna, Liquid Crystalline Polymers, Cambridge University, 2006.

[6] X. Zhao, K. Nair, P. Yung, G. Barber, S. Gray, Y. S. Kim and A. Maliqi, U.S. Patent, US9206300 B2,2015.

[7] A. A. Handlos and D. G. Baird, "Processing and Associated Properties of In Situ Composites Based on Thermotropic Liquid Crystalline Polymers and Thermoplastics", Journal of Macromolecular Science, Part C, vol.35, pp 183-238, 1995.

[8] J. Lange and Y. Wyser, "Recent innovations in barrier technologies for plastic packaging - a review", Packaging Technology and Science, vol.16, pp 149-158, 2003.

[9] R. Lusignea, "High-barrier packaging with liquid crystal polymers", Tappi Journal, vol.80, pp 205-212, 1997.

[10] C. Qian, C. Mansfield and D. G. Baird, "Thermotropic liquid crystalline polymers and their fiber reinforced composites for hydrogen storage applications", Annual Technical Conference, edited by (Society of Plastics Engineers, 2014), vol.

[11] R. W. Gray, D. G. Baird and J. H. Bøhn, "Effects of Processing Conditions on Short TLCP Fiber Reinforced FDM Parts", Rapid Prototyping Journal, vol.4, pp 14-25, 1998. 
[12] D. G. Baird, M. Ansari, C. D. Mansfield and C. Qian, "Generation of thermotropic liquid crystalline polymer thermoplastic composite filaments and their processing in fused filament fabrication", 251st ACS National Meeting, edited by (American Chemical Society, 2016), vol.

[13] F. J. Vallejo, I. Iribarren, J. I. Eguiazabal and J. Nazabal, "Structure and mechanical properties of blends of two thermotropic copolyesters", Polymer Engineering and Science, vol.42, pp 1686-1693, 2002.

[14] S. Kenig, M. T. DeMeuse and M. Jaffe, "Properties of blends containing two liquid crystalline polymers", Polymers for Advanced Technologies, vol.2, pp 25-30, 1991.

[15] F. J. Vallejo, J. I. Eguiazábal and J. Nazábal, "Blends of a thermotropic copolyester and a thermotropic copoly(ester-amide): structure and mechanical properties", Polymer, vol.42, pp 9593-9599, 2001.

[16] F. J. Vallejo, J. I. Eguiazabal and J. Nazabal, "Blends of two thermotropic liquid crystalline polymers: the influence of reactions during injection molding on the phase structure and the mechanical behavior", Polymer Engineering and Science, vol.41, pp 1115-1123, 2001.

[17] M. Garcia, J. I. Eguiazabal and J. Nazabal, "Miscibility level and mechanical characterization of blends of two liquid-crystalline polymers based on p-hydroxybenzoic acid", Journal of Polymer Science Part B: Polymer Physics, vol.41, pp 1022-1032, 2003.

[18] Y. G. Lin and H. H. Winter, "Rheology of phase-separated blends of two thermotropic liquid-crystalline copolyesters", Polymer Engineering and Science, vol.32, pp 773-776, 1992.

[19] T.-T. Hsieh, C. Tiu, G. P. Simon and R. Yu Wu, "Rheology and miscibility of thermotropic liquid crystalline polymer blends", Journal of Non-Newtonian Fluid Mechanics, vol.86, pp 15-35, 1999.

[20] M. A. McLeod and D. G. Baird, "The crystallization behavior of blends of thermotropic liquid crystalline polymers", Polymer, vol.40, pp 3743-3752, 1999.

[21] C. M. McCullagh, J. Blackwell and A. M. Jamieson, "Transesterification in Blends of Wholly Aromatic Thermotropic Copolyesters", Macromolecules, vol.27, pp 2996-3001, 1994.

[22] D. J. Blundell, "The nature of crystallites in solidified, rigid-chain, liquid crystal polymers", Polymer, vol.23, pp 359-364, 1982. 
[23] L. Yang, R. H. Somani, I. Sics, B. S. Hsiao, R. Kolb, H. Fruitwala and C. Ong, "ShearInduced Crystallization Precursor Studies in Model Polyethylene Blends by in-Situ RheoSAXS and Rheo-WAXD", Macromolecules, vol.37, pp 4845-4859, 2004.

[24] R. R. Lagasse and B. Maxwell, "An experimental study of the kinetics of polymer crystallization during shear flow", Polymer Engineering and Science, vol.16, pp 189-199, 1976.

[25] S. Vleeshouwers and H. E. H. Meijer, "A rheological study of shear induced crystallization", Rheologica Acta, vol.35, pp 391-399,

[26] A. Nogales, B. S. Hsiao, R. H. Somani, S. Srinivas, A. H. Tsou, F. J. Balta-Calleja and T. A. Ezquerra, "Shear-induced crystallization of isotactic polypropylene with different molecular weight distributions: in situ small- and wide-angle X-ray scattering studies", Polymer, vol.42, pp 5247-5256, 2001.

[27] Y. G. Lin and H. H. Winter, "Formation of high melting crystal in a thermotropic aromatic copolyester", Macromolecules, vol.21, pp 2439-2443, 1988.

[28] Y. G. Lin, H. H. Winter and G. Lieser, "Equibiaxial extension of a thermotropic liquidcrystalline polymer Flow induced orientation, relaxation of orientation, and strain recovery", Liquid Crystals, vol.3, pp 519-529, 1988. 


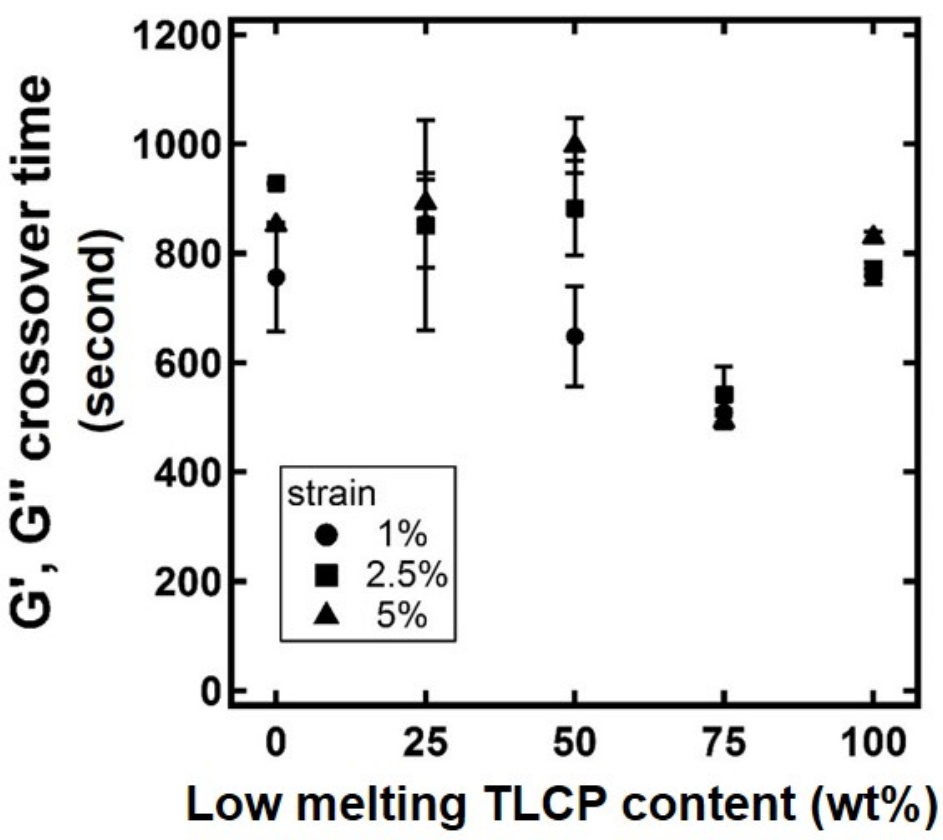

